\title{
PERFORMANCE ASSESSMENT OF A 2 DOF GYROSCOPIC WAVE ENERGY CONVERTER
}

\author{
Alessandro Battezzato \\ Center for Space Human Robotics, Istituto Italiano di Tecnologia, Torino, Italy \\ Giovanni Bracco, Ermanno Giorcelli, Giuliana Mattiazzo \\ Department of Mechanical and Aerospace Engineering, Politecnico di Torino, Torino, Italy \\ e-mail: giovanni.bracco@polito.it
}

\begin{abstract}
Wave Power is one of the most investigated energy sources today. So far, several devices have been tested and built up to the pre-commercial stage. ISWEC (Inertial Sea Wave Energy Converter) has developed at the Politecnico di Torino, exploiting gyroscopes to extract wave energy. It allows power extraction without using any moving part immersed into water. The previous version of ISWEC presented 1 DOF (Degree Of Freedom), therefore requiring alignment of the device to the incoming wave; this paper describes a novel version of ISWEC, with 2 DOFs and consequently able to absorb power from every wave direction. The kinematics and the dynamics of the device are investigated, in order to compare the $1 \mathrm{DOF}$ and the $2 \mathrm{DOF}$ architectures from the point of view of the extracted power. The resulting simulations show that the 1 DOF prototype is more efficient when aligned with the incoming wave, while the behavior of the 2 DOF device is substantially independent of the wave direction. Such a difference of the performances is quantified and discussed along with considerations on the design and realization of the full-scale prototype.
\end{abstract}

Keywords: gyroscope, wave power, flywheel, hydrodynamics, renewable energy

\section{Introduction}

Ocean is a wide source of different kinds of energies such as tides, marine streams, salinity gradients, temperature gradients and wave power. Wave power is the discipline studying the extraction of energy from sea waves. Until now, wave power is still a developing technology, even though research on sea power exploitation began in the early stages of the 19th century. At the time of writing, the field is growing with great interest from the big players of industry (Chen et al., 2013). Consistent investments have been made on the sector and several devices already reached the pre-commercial stage.

The Wave Energy Converters (WECs) proposed until now exploit different physical principles. This results in a variety of morphologies and sizes of the devices. WECs can be classified according to their position with respect to the sea (floating, tethered or submerged) and to their dimensions compared to the incoming wave: point absorbers, having dimensions negligible with respect to the wave length, attenuators, with their length comparable to the wave length, and terminators, having width comparable with the wave length. WECs can also be classified according to the working principle: Oscillating Water Columns (OWC), oscillating bodies and overtopping devices (Falcão, 2010; Torre-Enciso et al., 2010; Sorensen and Friis-Madsen, 2010; Le Crom et al., 2009; Lucas et al., 2007; Pizer et al., 2005).

The OWC is one of the first WECs ever investigated: since 1940 in Japan Masuda has designed and built several prototypes operating in real sea conditions. The OWC is a terminator composed of a submerged concrete or steel structure, open below the water level. The oscillating 
motion of the internal water level produced by the incident waves forces the air contained inside the chamber to flow through a turbine driving an electrical generator. Since the turbine must be bidirectional, the Wells turbine is the most used in this kind of WECs. Several realizations of OWC were completed, among which the Pico plant, Azores, Portugal in 1999 and the LIMPET plant in Islay island, Scotland in 2000. A floating version of the OWC was realized by the project OSPREY in UK, 2005.

The class of oscillating bodies includes floating devices and submerged devices. Dexawave (DK) and Pelamis (UK) are two floating devices exploiting the wave induced relative motion of two or several floating bodies to produce energy. In the joints connecting the bodies, there is an energy conversion system composed of hydraulic rams acted by the relative motion of the bodies, a hydraulic circuit to stabilize the oil flow coming from the alternate motion of the rams and a hydraulic motor driving the electric generator. The energy conversion system is usually referred to as Power Take Off - PTO. Another oscillating body device is the AWS (UK). It is a submerged device composed by a fixed part moored to the seabed and a vertical sliding cap separated with an air chamber. As waves approach the device, the hydrostatic pressure on the top of the cap changes making it oscillate vertically and acting linear electric generators. The stiffness of the air chamber can be changed to tune the device on the incoming wave.

The last category of overtopping devices is represented mainly by terminators: they convey waves on a slope and collect water on an elevated reservoir: the production of energy is performed by returning the water to the sea level through a low-head turbine coupled with a generator. An example of realization of the machine is represented by the Danish project Wave Dragon.

One of the problems to be solved in a WEC is the so called "reaction problem" (French, 2006): in order to extract power from the sea surface with a force, a reaction to that force must be provided. In his analysis, French highlighted that the reaction force can be given in four different ways: reacting on a large structure bigger than the wavelength and therefore hydrodynamically stable, reacting to the seabed, reacting on a mass that is part of the WEC and reacting against a part of the sea. Except for the third choice, the other possibilities imply the use of mechanical parts in motion while immersed into sea water or spray and thus the need of expensive solutions such as reliable seals and corrosion protection techniques. In this article, the system ISWEC (Inertial Sea Wave Energy Converter) is proposed. ISWEC uses a gyroscope to create an internal inertial reaction able to harvest wave power without exposed mechanical parts. In fact, ISWEC externally presents as a monolithic float: the float rocks in reaction to the incoming wave and the gyroscopic system is sealed inside.

The rotor dynamics is a discipline continuously investigated due to its several applications (Ładyżyńska-Kozdraś and Koruba, 2012; Baranowski, 2013). The use of its inertial effects to harvest wave power was investigated on a 1 degree of freedom ISWEC device (Bracco, 2012; Bracco et al., 2010, 2011) proving its power absorption capacities. The 1 DOF device so far proposed and investigated is unidirectional, meaning that the system must be aligned with the incoming wave in order to absorb the maximum power, while the power absorption drastically decreases when diverging from this preferred direction. To overcome this problem, a novel 2 DOF ISWEC device has been derived: now the gyroscope can freely rotate about two orthogonal axes, in order to decrease and ideally neglect the influence of the wave direction. This paper deals with the investigation of the 2 DOF ISWEC system, in order to determine its power conversion capabilities and its multi-directionality effectiveness. To perform such analysis, a robotic approach is taken into account: first of all, the position of each linkage of the mechanism is described through the definition of a number of reference frames and proper DOFs, then the velocities are investigated and finally the dynamical equations are written according to the Lagrangian approach. Once the extracted power is analytically expressed, an optimization process is carried out in order to find values of the coefficients of the stiffness and damping elements of the ISWEC prototype maximizing the extracted power itself. Finally, a number 
of simulations have been launched, in order to investigate the theoretical effectiveness of the optimized 2 DOF prototype for the varying wave direction. The final results permit one to take important design considerations and proper choices towards the realisation of a full scale prototype of the ISWEC.

\section{ISWEC working principle}

As shown in Fig. 1, the ISWEC is a floating Wave Energy Converter externally appearing as a monolithic hull with a slack mooring to the sea bed.

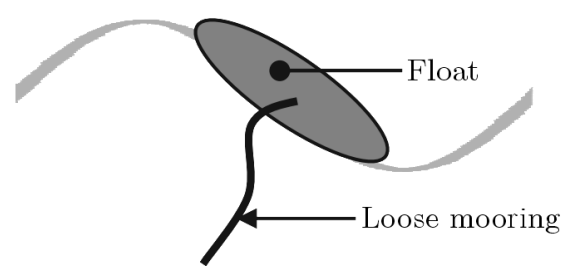

Fig. 1. ISWEC external view

Inside the ISWEC, there is a gyroscope that is carried on a 1 DOF or a 2 DOF (gimbal) structure. In the 2 DOF system, the flywheel is carried on a gimbal allowing the flywheel to precess with two degrees of freedom $\varepsilon$ and $\lambda$. The PTOs (Power-Take-Off, the device converting mechanical energy into electrical energy) in the representation of Fig. 2 are linear devices linked to the inner platform of the gimbal and the hull.

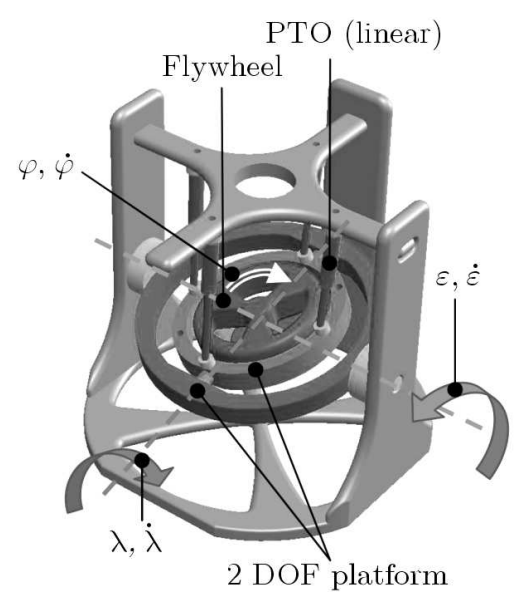

Fig. 2. The 2 DOF ISWEC

The working principle of the device can be summarized in three main steps:

- The waves tilt the buoy

- Due to stiffness of the PTO, a fraction of this rotation is transmitted to the inner platform of the gimbal perturbing the gyroscope

- Thanks to the inertial effect, the gyro starts its precession. By damping the precession and restoring the initial condition, the PTOs produce energy.

The job of the PTOs is both to link the gyro to the gimbal and to damp the motion of the gyro to extract energy. In Fig. 2, they are considered as four linear actuators but they can also be rotary motors. In the second case, they are coupled directly on the axis of motion to damp, their shaft on the mobile part and their carter on the still part. In this configuration, there are 
no restrains to the angles performed by the gimbal, and the gyro can freely rotate around the two axis. Such a system, as shown in the next Sections, is omni-directional.

On the other hand, in the 1 DOF version shown in Fig. 3, the system must be pointed towards the waves and in response to pitch motion of the float the systems precesses around the coordinate $\varepsilon$ driving in rotation a PTO. By controlling the PTO (Bracco et al., 2009) the extraction of energy from the waves is achieved.

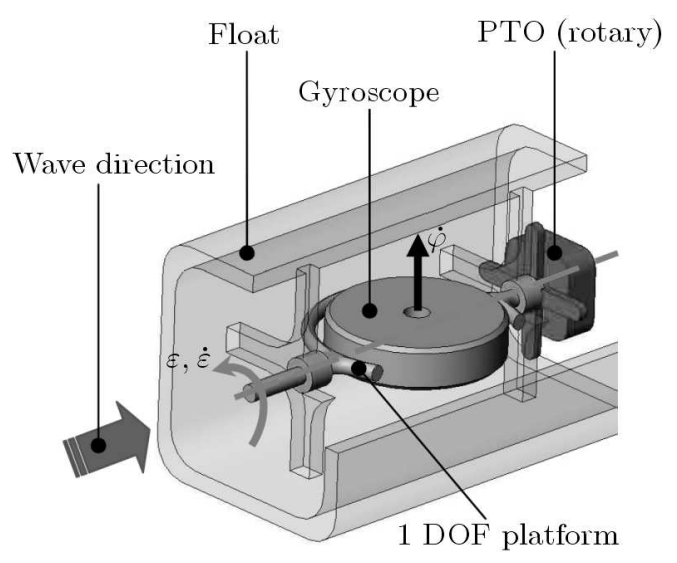

Fig. 3. The 1 DOF ISWEC

\subsection{Equations of motion}

The first step to study the kinematical and dynamical behavior of the ISWEC architecture consists in assigning a coordinate reference system to each body composing the system. In fact, given a reference system attached to a body, then the position and orientation of the body are completely defined if the position and orientation of the reference system are known. First of all, it is necessary to place a fixed reference system, referred to as $C S_{0}$, whose axis $z_{0}$ is directed vertically. The direction from which the wave is arriving is identified by the axis $y_{0^{\prime}}$ belonging to $C S_{0^{\prime}}$, a reference system obtained from $C S_{0}$ through rotation of the angle $\beta$ about $z_{0} \equiv z_{0^{\prime}}$. The system $C S_{1}$ is associated with the frame which carries the gyroscope: it is obtained from $C S_{0}$ through rotation of the angle $\delta$ about the axis $x_{0^{\prime}}$ belonging to $C S_{0^{\prime}}$. Then, the flywheel is linked to the frame through a pair of rings. The reference system named as $C S_{2}$ is associated with the external ring: it can be obtained from $C S_{1}$ through rotation of the angle $\varepsilon$ about $y_{1} \equiv y_{2}$. On the other side, the reference system $C S_{3}$ is linked to the internal ring: it is obtained from $C S_{2}$ through rotation of the angle $\lambda$ about $x_{2} \equiv x_{3}$. Finally, the reference system $C S_{4}$ is associated with the flywheel: it is obtained from $C S_{3}$ through rotation of the angle $\varphi$ about $z_{3} \equiv z_{4}$. The coordinate reference systems are represented in Fig. 4.

(a)

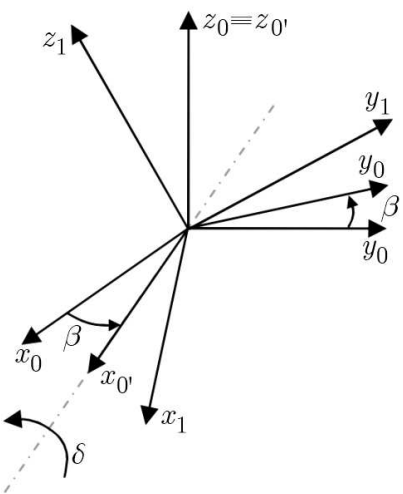

(b)

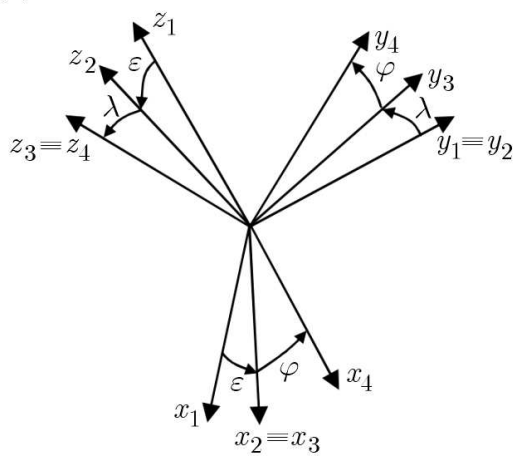

Fig. 4. Representation of the coordinate reference systems: (a) from $C S_{0}$ to $C S_{1}$, (b) from $C S_{1}$ to $C S_{4}$ 
Once the reference systems associated with the whole mechanism have been defined, then it is possible to study the kinematics of the system. Given the previously introduced reference systems, the following rotation matrices between successive reference systems can be expressed, where ${ }^{j} \mathbf{A}_{i}$ is the matrix that permits one to pass from a generic $C S_{i}$ to $C S_{j}$

$$
\begin{aligned}
& { }^{0} \mathbf{A}_{0^{\prime}}=\operatorname{rot}\left(z_{0}, \beta\right)=\left[\begin{array}{ccc}
c \beta & -s \beta & 0 \\
s \beta & c \beta & 0 \\
0 & 0 & 1
\end{array}\right] \\
& { }^{0^{\prime} \mathbf{A}_{1}}=\operatorname{rot}\left(x_{0^{\prime}}, \delta\right) \operatorname{rot}\left(z_{1},-\beta\right)=\left[\begin{array}{ccc}
1 & 0 & 0 \\
0 & c \delta & -s \delta \\
0 & s \delta & c \delta
\end{array}\right]\left[\begin{array}{ccc}
c \beta & s \beta & 0 \\
-s \beta & c \beta & 0 \\
0 & 0 & 1
\end{array}\right]=\left[\begin{array}{ccc}
c \beta & s \beta & 0 \\
-s \beta c \delta & c \beta c \delta & -s \delta \\
-s \beta s \delta & c \beta s \delta & c \delta
\end{array}\right] \\
& { }^{2} \mathbf{A}_{3}=\operatorname{rot}\left(x_{2}, \lambda\right)=\left[\begin{array}{ccc}
1 & 0 & 0 \\
0 & c \lambda & -s \lambda \\
0 & s \lambda & c \lambda
\end{array}\right]
\end{aligned}
$$

In the previous (2.1) and in the following, letters $c$ and $s$ stand for cosine and sine respectively. Moreover, if ${ }^{j} \mathbf{A}_{i}$ in (2.1) is the rotation matrix between $C S_{i}$ and $C S_{j}$, then the transposes of (2.1) give the ${ }^{i} \mathbf{A}_{j}$ rotation matrices between $C S_{j}$ and $C S_{i}$.

The analysis of the velocities and accelerations of the ISWEC system is limited to the study of the angular terms; in fact, given the previous description of the whole architecture, no linear translations are taken into account in the modeling of the system. In the following equations, the notation $\mathbf{i}_{i}, \mathbf{j}_{i}$ and $\mathbf{k}_{i}$ refers to the unit vectors respectively directed along the axes $x_{i}, y_{i}$ and $z_{i}$ of the system $C S_{i}$. Moreover, the apex $i$ that precedes a generic vector ${ }^{i} \mathbf{a}$ indicates that vector $\mathbf{a}$ is expressed in the reference system $C S_{i}$. When not specified, in the following, the vectors are intended as expressed in $C S_{0}$. The angular velocity $\boldsymbol{\omega}_{i}$ of the $i$-th reference system is

$$
\begin{array}{rlrl}
{ }^{1} \boldsymbol{\omega}_{1} & =\dot{\delta}^{1} \mathbf{A}_{0^{\prime}}{ }^{\prime} \mathbf{i}_{0^{\prime}} & { }^{2} \boldsymbol{\omega}_{2}={ }^{2} \mathbf{A}_{1}\left(\dot{\varepsilon}^{1} \mathbf{j}_{1}+{ }^{1} \boldsymbol{\omega}_{1}\right) \\
{ }^{3} \boldsymbol{\omega}_{3}={ }^{3} \mathbf{A}_{2}\left(\dot{\lambda}^{2} \mathbf{i}_{2}+{ }^{2} \boldsymbol{\omega}_{2}\right) & { }^{3} \boldsymbol{\omega}_{4}=\dot{\varphi}^{3} \mathbf{k}_{3}+{ }^{3} \boldsymbol{\omega}_{3}
\end{array}
$$

Time derivatives of (2.2) lead to the angular accelerations that are here reported

$$
\begin{aligned}
{ }^{1} \dot{\boldsymbol{\omega}}_{1}=\ddot{\delta}^{1} \mathbf{A}_{0^{\prime}}{ }^{\prime} \mathbf{i}_{0^{\prime}} & { }^{2} \dot{\boldsymbol{\omega}}_{2}={ }^{2} \mathbf{A}_{1}\left(\ddot{\varepsilon}^{1} \mathbf{j}_{1}+\dot{\varepsilon}^{1} \boldsymbol{\omega}_{1} \times{ }^{1} \mathbf{j}_{1}+{ }^{1} \dot{\boldsymbol{\omega}}_{1}\right) \\
{ }^{3} \dot{\boldsymbol{\omega}}_{3}={ }^{3} \mathbf{A}_{2}\left(\ddot{\lambda}^{2} \mathbf{i}_{2}+\dot{\lambda}^{2} \boldsymbol{\omega}_{2} \times{ }^{2} \dot{\mathbf{i}}_{2}+{ }^{2} \dot{\boldsymbol{\omega}}_{2}\right) & { }^{3} \dot{\boldsymbol{\omega}}_{4}=\ddot{\varphi}^{3} \mathbf{k}_{3}+\dot{\varphi}^{3} \boldsymbol{\omega}_{3} \times{ }^{3} \mathbf{k}_{3}+{ }^{3} \dot{\boldsymbol{\omega}}_{3}
\end{aligned}
$$

Each velocity vector in (2.2) and (2.3) is expressed in its own reference system, except for $\boldsymbol{\omega}_{4}$ and its time derivative, which are expressed in $C S_{3}$. This is due to the axial symmetry hypothesis of link 4, as explained in the following.

The successive step consists in the dynamical analysis of the ISWEC system; the reference systems previously defined are supposed to be central, i.e. the inertial tensor of each body with respect to the body reference system is diagonal and the origin of the coordinate system coincides with the center of mass. Hence, the inertial tensor of the $i$-th body with respect to the $i$-th coordinate system is

$$
{ }^{i} \mathbf{I}_{i}=\left[\begin{array}{ccc}
I_{x i} & 0 & 0 \\
0 & I_{y i} & 0 \\
0 & 0 & I_{z i}
\end{array}\right]
$$

Each tensor is diagonal and constant only when expressed in its coordinate system; the only exception is the flywheel that presents a diagonal inertial tensor also when expressed in $C S_{3}$ due to its axial symmetry 


$$
{ }^{3} \mathbf{I}_{4}={ }^{3} \mathbf{A}_{4}{ }^{4} \mathbf{I}_{4}{ }^{3} \mathbf{A}_{4}^{\top}=\left[\begin{array}{ccc}
c \varphi & -s \varphi & 0 \\
s \varphi & c \varphi & 0 \\
0 & 0 & 1
\end{array}\right]\left[\begin{array}{ccc}
I_{x 4} & 0 & 0 \\
0 & I_{y 4} & 0 \\
0 & 0 & I_{z 4}
\end{array}\right]\left[\begin{array}{ccc}
c \varphi & s \varphi & 0 \\
-s \varphi & c \varphi & 0 \\
0 & 0 & 1
\end{array}\right]=\left[\begin{array}{ccc}
I_{x y 4} & 0 & 0 \\
0 & I_{x y 4} & 0 \\
0 & 0 & I_{z 4}
\end{array}\right]
$$

where $I_{x 4}=I_{y 4}=I_{x y 4}$. To solve the dynamics of the system, the Lagrangian approach can be applied. To manage the problem, it is fundamental to properly define the unknowns of the system. In fact, given the previously introduced angles, it is important to notice that some of them will be considered as given, while the other are unknowns and have to be calculated. First of all, in the current model, the sea conditions are imposed: thus, the angle $\delta$ and its time derivatives as well as angle $\beta$ are known. Also the angle of rotation of the flywheel $\varphi$ and its derivatives are supposed to be imposed by the motor: in particular, the flywheel rotates at a constant velocity $\dot{\varphi}$, which leads to $\ddot{\varphi}=0$. Thus, the only unknowns in the current model of the system are the two passive angles $\varepsilon$ and $\lambda$ and their time derivatives. It is important to point out that elastic and viscous elements are applied to the revolute joint between the frame and the external ring described by the rotational stiffness constant and the damping ratio $k_{\varepsilon}$ and $c_{\varepsilon}$. Analogously, the elastic and viscous elements are also applied to the revolute joint between the external and the internal ring: the rotational stiffness constant is $k_{\lambda}$ and the damping ratio is $c_{\lambda}$. Moreover, $\varepsilon_{0}$ and $\lambda_{0}$ are the values of the angles in the two rotational joints where the torque springs give the null response, while $\dot{\varepsilon}_{0}$ and $\dot{\lambda}_{0}$ are the rotational velocities where the torque dampers give the zero torque.

In order to write the Lagrangian equations of the system, the generalized coordinates $q_{i}$ have to be chosen: they are the angles $\varepsilon$ and $\lambda$. The generic Lagrangian equation for a dynamic system is the following

$$
\frac{d}{d t}\left(\frac{\partial T}{\partial \dot{q}_{i}}\right)-\frac{\partial T}{\partial q_{i}}+\frac{\partial V}{\partial q_{i}}+\frac{\partial D}{\partial q_{i}}=0
$$

where $T$ is the kinetic energy of the system, $V$ is the potential energy and $D$ is the dissipation function. In the current ISWEC model, $T$ has only rotational terms, $V$ takes into account the presence of the elastic elements, while the viscous terms are included in the dissipation function $D$. In fact it is

$$
T=\frac{1}{2}{ }^{1} \boldsymbol{\omega}_{1}^{\top 1} \mathbf{I}_{1}{ }^{1} \boldsymbol{\omega}_{1}+\frac{1}{2}{ }^{2} \boldsymbol{\omega}_{2}^{\top 2} \mathbf{I}_{2}{ }^{2} \boldsymbol{\omega}_{2}+\frac{1}{2}{ }^{3} \boldsymbol{\omega}_{3}^{\top 3} \mathbf{I}_{3}{ }^{3} \boldsymbol{\omega}_{3}+\frac{1}{2}{ }^{3} \omega_{4}^{\top 3}{ }^{3} \mathbf{I}_{4}{ }^{3} \omega_{4}
$$

and

$$
V=\frac{k_{\varepsilon}}{2}\left(\varepsilon-\varepsilon_{0}\right)^{2}+\frac{k_{\lambda}}{2}\left(\lambda-\lambda_{0}\right)^{2} \quad D=\frac{c_{\varepsilon}}{2}\left(\dot{\varepsilon}-\dot{\varepsilon}_{0}\right)^{2}+\frac{c_{\lambda}}{2}\left(\dot{\lambda}-\dot{\lambda}_{0}\right)^{2}
$$

If, on the other hand, the PTO components are four linear parts like in the example shown in Fig. 2, previous equations (2.8) change slightly. Given $\bar{k}_{\varepsilon}$ and $\bar{c}_{\varepsilon}$ the linear spring constant and damping ratio of the elements connecting the frame to the external ring, and $\bar{k}_{\lambda}$ and $\bar{c}_{\lambda}$ the linear spring constant and damping ratio of the elements connecting the frame to the internal ring, then equations (2.8) can be substituted by the following $(2.9)_{1}$ and $(2.9)_{2}$, respectively

$$
\begin{aligned}
& V=\frac{\bar{k}_{\varepsilon}}{2}\left[\left(r_{1 \varepsilon}-r_{1 \varepsilon_{-} 0}\right)^{2}+\left(r_{2 \varepsilon}-r_{2 \varepsilon_{-} 0}\right)^{2}\right]+\frac{\bar{k}_{\lambda}}{2}\left[\left(r_{1 \lambda}-r_{1 \lambda_{-} 0}\right)^{2}+\left(r_{2 \lambda}-r_{2 \lambda_{-} 0}\right)^{2}\right] \\
& D=\frac{\bar{c}_{\varepsilon}}{2}\left[\left(\dot{r}_{1 \varepsilon}-\dot{r}_{1 \varepsilon_{-} 0}\right)^{2}+\left(\dot{r}_{2 \varepsilon}-\dot{r}_{2 \varepsilon_{-} 0}\right)^{2}\right]+\frac{\bar{c}_{\lambda}}{2}\left[\left(\dot{r}_{1 \lambda}-\dot{r}_{1 \lambda_{-} 0}\right)^{2}+\left(\dot{r}_{2 \lambda}-\dot{r}_{2 \lambda_{-} 0}\right)^{2}\right]
\end{aligned}
$$

where $r_{i j}=\left|\mathbf{p}_{i j}-\mathbf{q}_{i j}\right|$, with $i=1,2$, and $j=\varepsilon, \lambda$. The vector $\mathbf{p}_{i j}$ gives the position of the connection point between the ring and the corresponding PTO element; the vector $\mathbf{q}_{i j}$ gives 
the position of the connection point between the same PTO element and the external frame. The terms $r_{i j_{-} 0}$ and $\dot{r}_{i j_{0} 0}$ express the linear offset, respectively associated with the elastic and damping effect. Thus, two Lagrangian equations (2.6), written with respect to $q_{1}=\varepsilon$ and $\lambda, \dot{\lambda}$ are

$$
E_{1} \ddot{\varepsilon}+E_{2} \dot{\varepsilon}+E_{3} \dot{\varepsilon} \dot{\lambda}+E_{4} \dot{\lambda}+E_{5}=0 \quad L_{1} \ddot{\lambda}+L_{2} \dot{\lambda}+L_{3} \dot{\varepsilon}^{2}+L_{4} \dot{\varepsilon}+L_{5}=0
$$

It is noteworthy that the coefficients are not constants but functions of the angles $\varepsilon$ and $\lambda$, too. The system composed by equations (2.10) can be solved through a numerical software and the trend of the angles $\varepsilon$ and $\lambda$, as well as the kinematics of the whole system can be calculated from the initial conditions. Afterwards, also some dynamical outputs can be calculated; for example the torque $T_{\varphi}$ that the motor has to apply to the flywheel in order to maintain the constant velocity $\dot{\varepsilon}$ is

$$
T_{\varphi}=\left(\mathbf{I}_{4} \dot{\boldsymbol{\omega}}_{4}+\boldsymbol{\omega}_{4} \times \mathbf{I}_{4} \boldsymbol{\omega}_{4}\right) \cdot \mathbf{k}_{3}
$$

At the same time, the torque actions that are exerted on the flywheel along the joint axes, where the elastic and viscous elements are applied, are

$$
T_{\varepsilon}=-c_{\varepsilon}\left(\dot{\varepsilon}-\dot{\varepsilon}_{0}\right)-k_{\varepsilon}\left(\varepsilon-\varepsilon_{0}\right) \quad T_{\lambda}=-c_{\lambda}\left(\dot{\lambda}-\dot{\lambda}_{0}\right)-k_{\lambda}\left(\lambda-\lambda_{0}\right)
$$

\subsection{The small scale prototypes}

Most of the experimental work relative to the 1 DOF ISWEC has been carried out on the small scale prototype shown in Fig. 5 mainly at the Politecnico di Torino but using test facilities of the Univertities of Edinburgh and Naples. The prototype has a rated power of $2.2 \mathrm{~W}$ (Bracco, 2010), its flywheel has a diameter of $180 \mathrm{~mm}$, a moment of inertia of $0.0174 \mathrm{kgm}^{2}$ and spins at $2000 \mathrm{rpm}$.

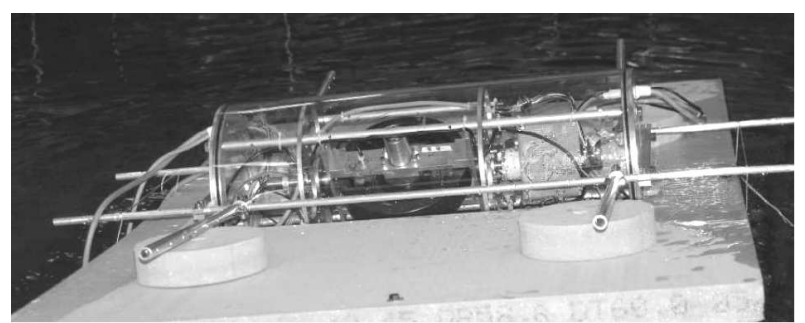

Fig. 5. The 1 DOF prototype in the wave tank at the Institute of Energy Systems of the University of Edinburgh, UK

The 1 DOF prototype has been designed to exploit the waves produced from the wave tank at the University of Edinburgh. The reference frequency of the wave tank when producing regular waves is $1 \mathrm{~Hz}$ (Taylor et al., 2003). In order to understand which angular motions are transferred to the float, a preliminary hydrodynamic analysis based on previous experimental works has been carried out, suggesting that the float will rock with an amplitude of motion equal to $2 \mathrm{deg}$. That analysis was actually verified one year later with the tank tests (Bracco et al., 2010).

In order to compare the 1 DOF and the 2 DOF device, the 2 DOF prototype shown in Fig. 6 has been built with the same inertial characteristics.

Apart from the mechanical architecture, the main difference between the two devices stands in the PTO. The 1 DOF device uses an electric PTO controlled by a commercial driver, whereas the 2 DOF prototype has pneumatic PTOs simulators coupled with springs, in which the action is regulated by pneumatic resistances put in the inlet of the chambers. The pneumatic equipment has been chosen because of the lack of commercially available linear generators of the required size. In any case at these small scales, it is common practice to damp the produced energy while concentrating on evaluating the amount of such energy to make full scale production analysis. 


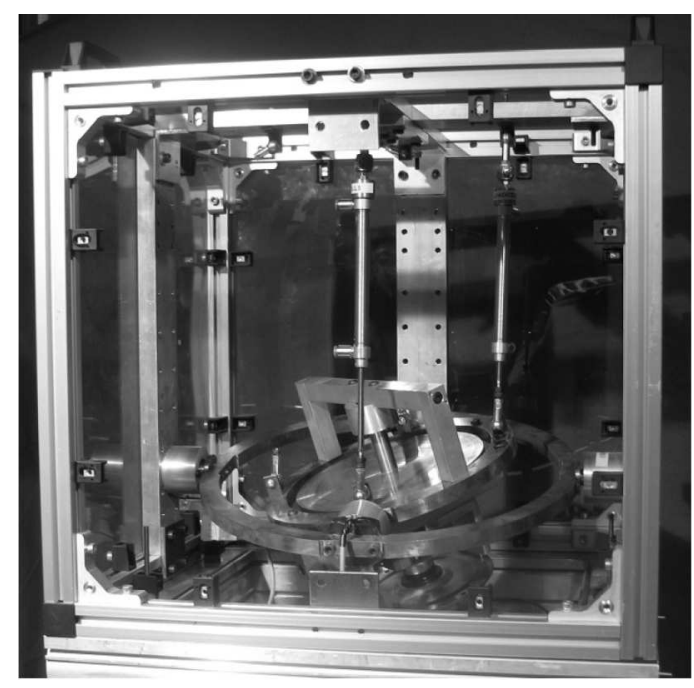

Fig. 6. The 2 DOF prototype model

\section{Results and simulations}

A number of simulations has been performed in order to study the system behavior and to optimize some design parameters. The goal of this optimization process for the 2 DOF ISWEC is to maximize $P_{d}$, the average power extracted from the system by the dampers evaluated in steady-state conditions. Accordingly with the 1 DOF tests, in the following simulations, the sea wave is described by the equation $\delta=\delta_{0} \sin (\omega t)$, where $\delta_{0}=2$ deg and the frequency $\omega$ is equal to $1 \mathrm{~Hz}$. While the inertial terms of the rings are neglected, the flywheel that rotates at a constant rotational velocity of $\dot{\varphi}=2000 \mathrm{rpm}$ has $I_{x y 4}=0.0166 \mathrm{kgm}^{2}$ and $I_{z 4}=0.0174 \mathrm{kgm}^{2}$.

Moreover, the elastic and viscous offset constants $\varepsilon_{0}$ and $\lambda_{0}, \dot{\varepsilon}_{0}$ and $\dot{\lambda}_{0}$ that appear in the preceding equations are all set to zero. In addition, to guarantee the isotropy of the mechanism, the damping and spring coefficients about the two passive rotational joints are equal: thus, in the following it is $c_{\varepsilon}=c_{\lambda}=c$ and $k_{\varepsilon}=k_{\lambda}=k$. Hence, the first simulations are set in order to find the maximum of $P_{d}$ for varying $c$ and $k$. The numerical code used for the simulations is the MATLAB ODE45 implementing the Runge-Kutta algorithm with a variable step. The angle $\beta$ is set equal to $30 \mathrm{deg}$. The results of these simulations are plotted in Fig. 7 - the maximum of $P_{d}$ occurs at $c=0.11, k=22$. Hence, the maximization of the power extracted from the ISWEC system leads to these elastic and damping coefficients.

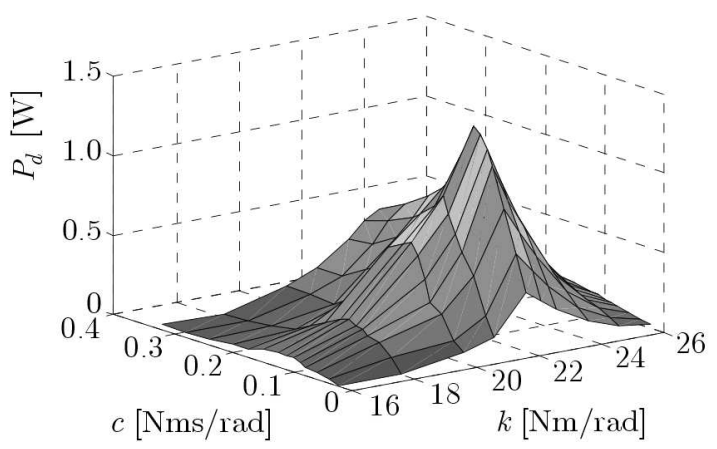

Fig. 7. Optimization of the coefficients $c$ and $k$

Finally, given the system with the optimized $c$ and $k$ coefficients, the steady-state periodic trend of the kinematic and dynamic variables of the system is reported. Figure 8a shows the input sea wave oscillation, i.e. the trend of angle $\delta$; the angle $\beta$ is set equal to $30 \mathrm{deg}$. Figure $8 \mathrm{~b}$ 
reports a quite sinusoidal trend of the angles $\varepsilon$ and $\lambda$ while Fig. $8 \mathrm{c}$ shows their time derivatives. All those variables proved to behave sinusoidally or quasi-sinusoidally on the field $c, k$ explored in this work. Figure 8d presents the torque actions $T_{\varepsilon}$ and $T_{\lambda}$, expressed in $(2.12)_{1}$ and $(2.12)_{2}$, respectively.

(a)
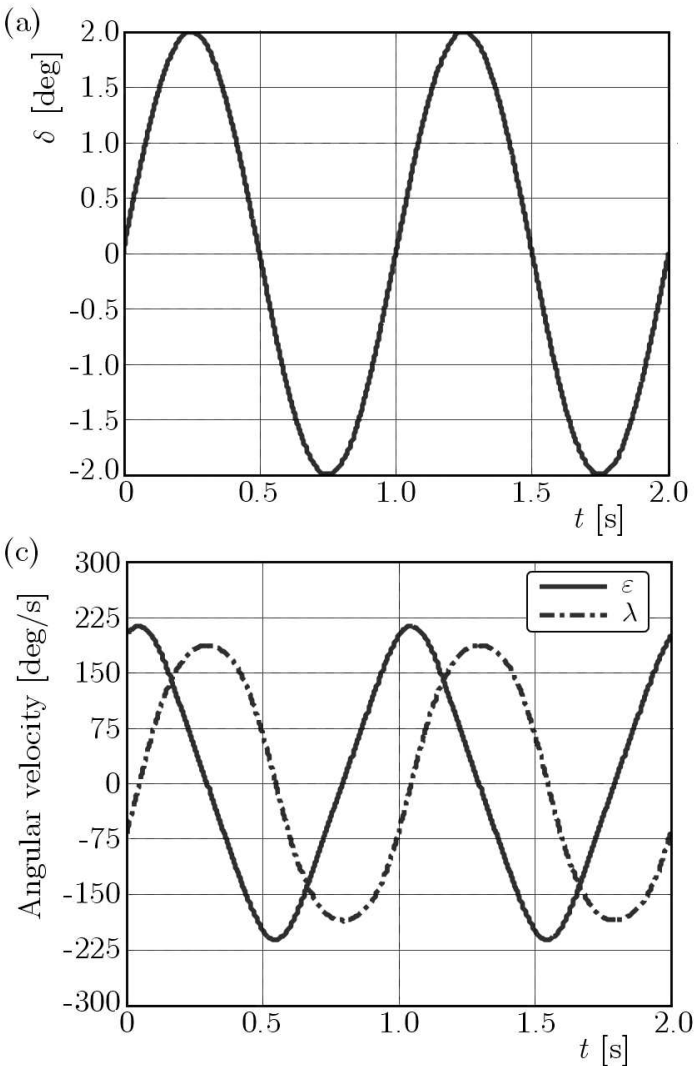

(b)

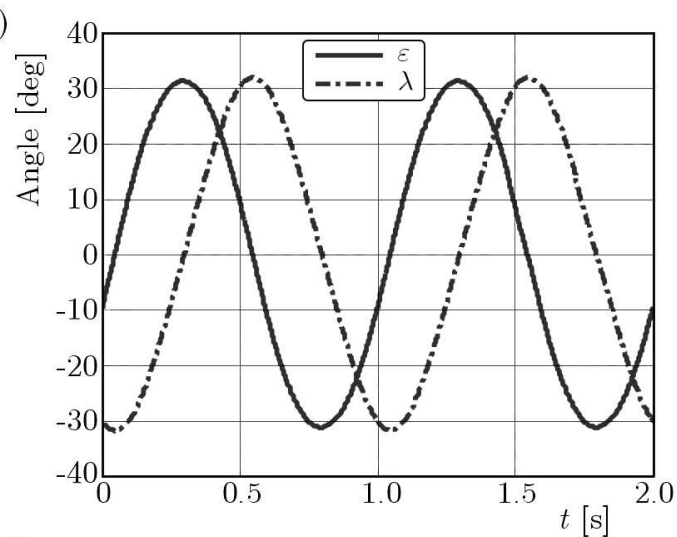

(d)

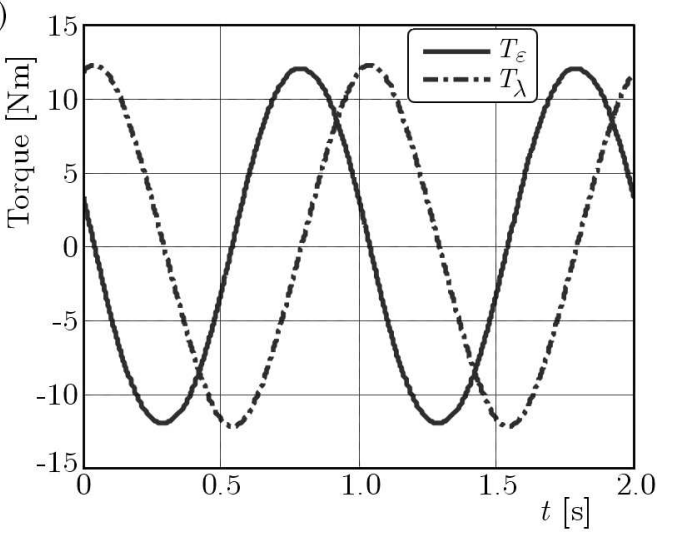

Fig. 8. (a) Time plot of the system input $\delta$; (b) time plot of position of the system 2 DOF; (c) time plot of velocity of the system 2 DOF; (d) time plot of the torque applied to the PTOs in the 2DOF system
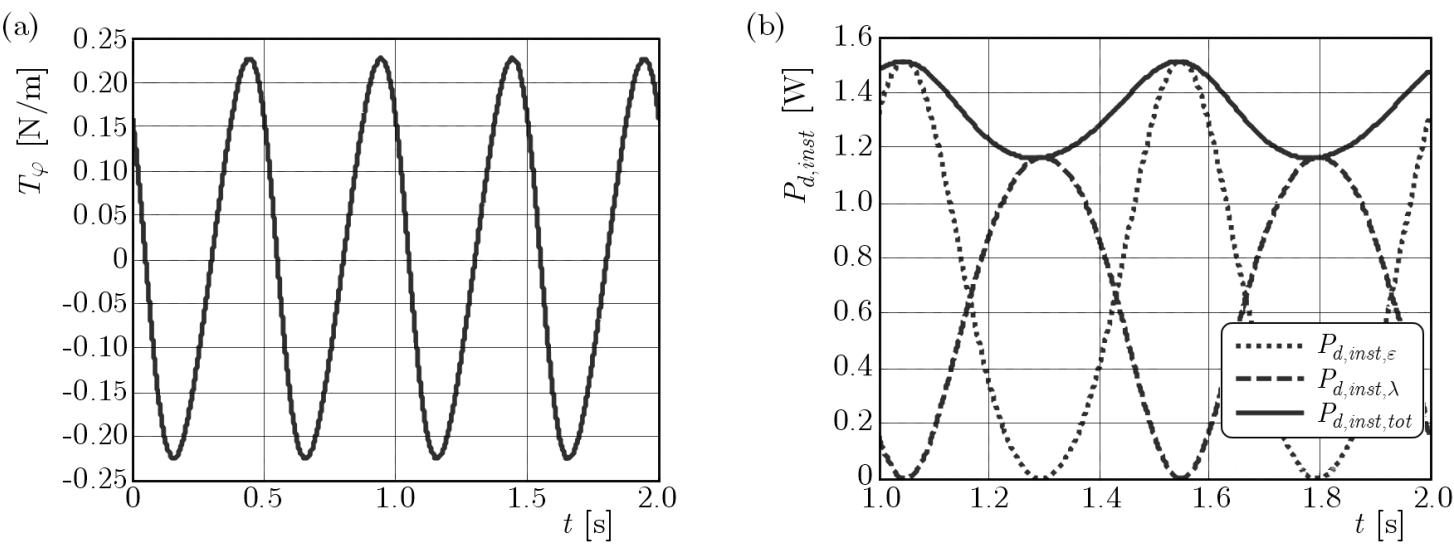

Fig. 9. (a) Time plot of the torque applied to the motor in the 2 DOF system (null mean value);

(b) time plot of the instantaneous power absorbed by the dampers in the 2 DOF system

The torque that the motor has to supply to the flywheel - calculated in $(2.11)$ - is shown in Fig. 9a. Finally, Fig. 9b reports the sinusoidal trend of the instantaneous power $P_{d, \text { inst }}$ whose value is the sum of the power terms $P_{d, i n s t, \varepsilon}$ and $P_{d, i n s t, \lambda}$ dissipated on the two revolute joints

$$
P_{d, i n s t, \varepsilon}=c_{\varepsilon}\left(\dot{\varepsilon}-\dot{\varepsilon}_{0}\right)^{2} \quad P_{d, i n s t, \lambda}=c_{\lambda}\left(\dot{\lambda}-\dot{\lambda}_{0}\right)^{2}
$$


These equations are valid for the rotational PTO, however similar expression can be formulated in the case of linear PTO components

$$
\begin{aligned}
& P_{d, i n s t, \varepsilon}=\bar{c}_{\varepsilon}\left[\left(\dot{r}_{1 \varepsilon}-\dot{r}_{1 \varepsilon_{-} 0}\right)^{2}+\left(\dot{r}_{2 \varepsilon}-\dot{r}_{2 \varepsilon_{-} 0}\right)^{2}\right] \\
& P_{d, i n s t, \lambda}=\bar{c}_{\lambda}\left[\left(\dot{r}_{1 \lambda}-\dot{r}_{1 \lambda_{-} 0}\right)^{2}+\left(\dot{r}_{2 \lambda}-\dot{r}_{2 \lambda_{-} 0}\right)^{2}\right]
\end{aligned}
$$

Figure 10a shows the time plot of the PTO shaft position in the 1 DOF system. The total instantaneous power output coming from the 2 DOF system is relatively smooth, whereas in the 1 DOF device it presents a peak about double with respect to the average value (see Fig. 10b). Therefore, the 1 DOF device could be mechanically simpler and cheaper to build, but the cost of the power electronics able to smooth such a variable power output could be relevant in the total economic balance.
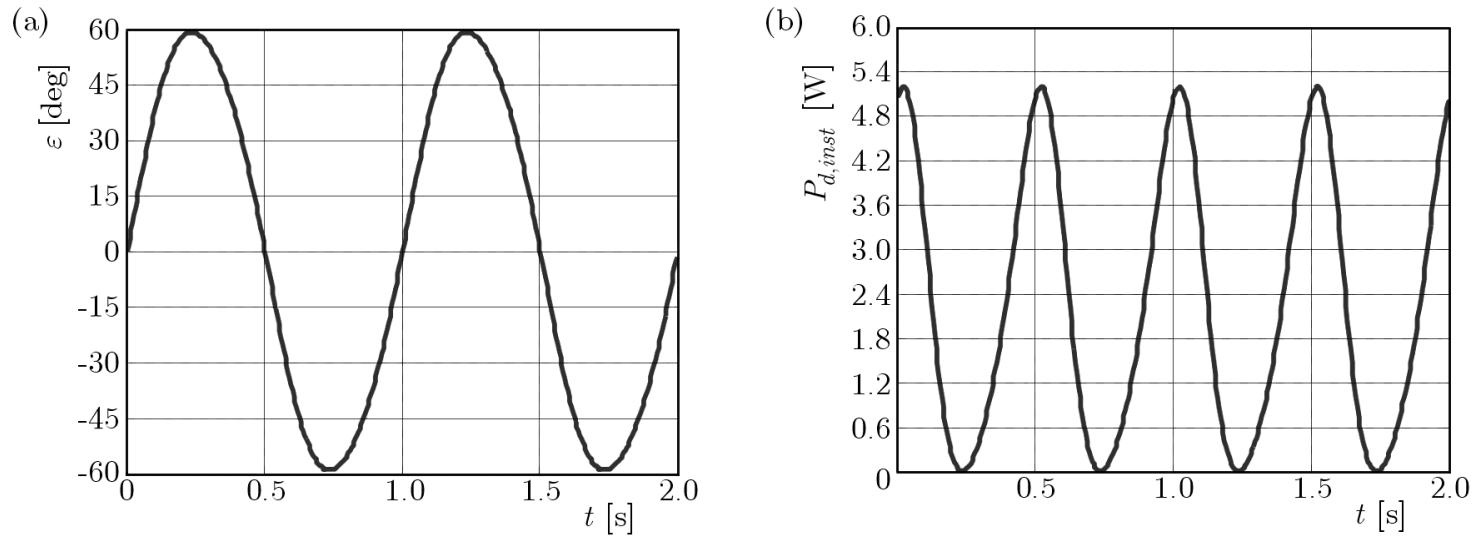

Fig. 10. (a) Angle of precession $\varepsilon$ of the 1 DOF system working in rated conditions; (b) time plot of the instantaneous power absorbed by the damper in the 1 DOF system working in rated conditions

Using the optimal $c$ and $k$ coefficients, the wave sea direction angle $\beta$ is varied. Thus, the influence of th angle $\beta$ on the trend of $P_{d}$ is reported in Fig. 11. It can be seen that the $2 \mathrm{DOF}$ model is practically insensitive to the wave direction from the $P_{d}$ point of view. On the other hand, the 1 DOF system shows a better power absorption when aligned with the incoming wave $(2.2 \mathrm{~W}$ against $1.32 \mathrm{~W})$, whereas the more waves come from the side, the smaller is the absorbed power. From this analysis, the 2 DOF device results are convenient in the field $52 \mathrm{deg}<\beta<128 \mathrm{deg}$.

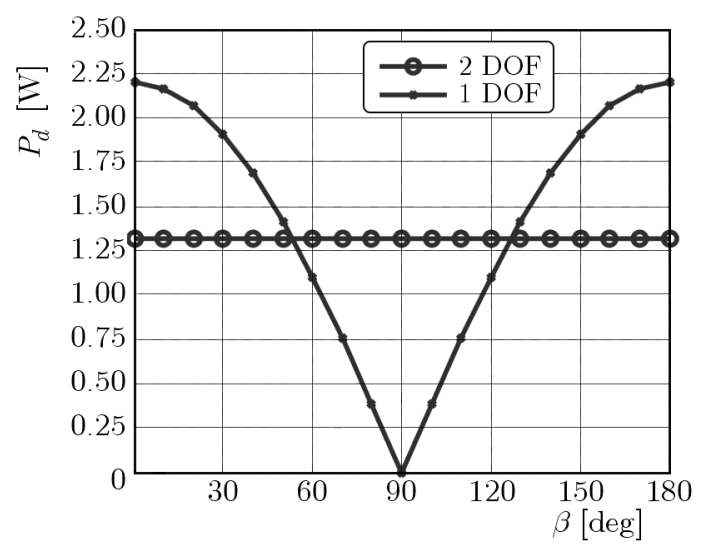

Fig. 11. Average absorbed power with respect to sea wave direction $\beta$ 


\section{Full scale considerations}

By scaling the results according to Froude, as it is common practice with floating WECs (Newman, 1977), the full scale parameters summarized in Table 1 are obtained. That scaling process has been done using the reference sea of Alghero, Italy, described through the Pierson-Moskowitz (Vicinanza et al., 2009) spectrum with the peak period equal to $6.7 \mathrm{~s}$ and a significant wave height equal to $1.19 \mathrm{~m}$ (2007 yearly average values). According to Froude and being the two prototypes designed to work at the $1 \mathrm{~Hz}$ wave, when they are compared to the real sea they are about 1:45 scaled $\left(6.7^{2}=44.9\right)$. Table 1 shows that the two prototypes are representative of a full scale system with power of the order of the MW. The gyroscope needed to harvest such power is relatively big if scaled directly from the prototype. However, this value can be split in different gyros in the case the unique disc is not feasible. The angles $\varepsilon$ and $\delta$ are not recalled in Table 1 because, according to Froude, they are the same between the scaled prototype and the real system (scale factor $=0$ ). The losses to maintain the gyro in rotation in the two prototypes are of the same order of magnitude of the produced power because of the small scale and the gyro spinning in the atmosphere. However, at the time of writing, a 1:8 scaled prototype with rated power $214 \mathrm{~W}$ is under construction. The prototype uses standard ball bearings and a vacuum chamber to reduce the gyro losses below 10\% of the produced power (Bracco et al., 2010), and from the preliminary analysis the same technology can be used in full scale devices to achieve effective power production.

Table 1. Froude scaling to full scale

\begin{tabular}{|l|c|c|c|}
\hline & $\begin{array}{c}\text { Froude scale } \\
\text { factor }\end{array}$ & Prototype & Full scale \\
\hline \hline Power (2 DOF) & 3.5 & $1.32 \mathrm{~W}$ & $0.81 \mathrm{MW}$ \\
\hline Power (1 DOF) & 3.5 & $2.2 \mathrm{~W}$ & $1.34 \mathrm{MW}$ \\
\hline Angular momentum & 4.5 & $3.48 \mathrm{kgm}^{2} \mathrm{rad} / \mathrm{s}$ & $1 \mathrm{E}+08 \mathrm{kgm}^{2} \mathrm{rad} / \mathrm{s}$ \\
\hline Gyro mass & 3 & $1.2 \mathrm{~kg}$ & $110 \mathrm{ton}$ \\
\hline Gyro speed & -0.5 & $2000 \mathrm{rpm}$ & $300 \mathrm{rpm}$ \\
\hline Wave period & 0.5 & $1 \mathrm{~s}$ & $6.7 \mathrm{~s}$ \\
\hline
\end{tabular}

\section{Conclusions}

In this paper, the 2 DOF ISWEC gyroscopic wave energy converter is proposed and analyzed in order to investigate its effectiveness with respect to similarly conceived 1 DOF ISWEC architecture. Such a comparison has been carried out in terms of capability of power extraction at variable direction of the incoming sea waves. In detail, once the kinematics and dynamics of the novel device have been solved, the spring and damping coefficients of the PTO have been investigated within the field of interest in order to maximize the absorbed power of the $2 \mathrm{DOF}$ prototype. Such an optimized configuration has been analyzed in the time domain and compared with the performance of the 1 DOF ISWEC with the same inertial characteristics. The 1 DOF system is more convenient in terms of the power output, producing $2.2 \mathrm{~W}$ against the $1.32 \mathrm{~W}$ of the 2 DOF device. However, the power output of the 2 DOF system is much more regular around its mean value, meaning less investments in power electronics smoothing systems. Moreover, the 2 DOF system proved to be truly omni-directional, whereas the 1 DOF system performs better only if aligned with the incoming wave. Future works will deal with the translation of these results into effective design considerations, which will be a guideline towards the full scale ISWEC prototype. 


\section{Acknowledgements}

The present work was realized in collaboration with ENEA on the agreement with the Ministero dello Sviluppo Economico "Accordo di Programma PAR 2013".

\section{References}

1. BARANOWSKi L., 2013, Equations of motion of a spin-stabilized projectile for flight stability testing, Journal of Theoretical and Applied Mechanics, 51, 1, 235-246

2. Bracco G., 2012, ISWEC: a Gyroscopic Wave Energy Converter, Lambert Academic Publishing, 9783848406524

3. Bracco G., Giorcelli E., Mattiazzo G., 2010, ISWEC: Design of a prototype model for wave tank test, Proceedings of the ESDA 2010, Istanbul, Turkey, ISBN: 9780791838778

4. Bracco G., Giorcelli E., Mattiazzo G., 2011, A gyroscopic mechanism for wave power exploitation, Mechanism and Machine Theory, pp.14, ISSN: 0094-114X, DOI: 10.1016/j.mechmachtheory.2011.05.012

5. Bracco G., Giorcelli E., Mattiazzo G., Pastorelli M., Taylor J.R.M., 2009, ISWEC: design of a prototype model with a gyroscope, Proceedings of the International Conference on Clean Electrical Power, ICCEP, Naples, Italy

6. Bracco G., Giorcelli E., Mattiazzo G., Poggi D., 2010, ISWEC: the use of gyroscopes to harvest wave power, Procedings of the Workshop on Oceans as a Source of Energy, Lisbon

7. Bracco G., Giorcelli E., Mattiazzo G., Poggi D., Taylor J., 2010, ISWEC: experimental tests on a small scale prototype model, Procedings of the 3rd International Conference on Ocean Energy, ICOE 2010, Bilbao, Spain

8. Chen Z., Yu H., Hu M., Meng G., Wen C,, 2013, A review of offshore wave energy extraction system, Advances in Mechanical Engineering

9. Cruz J., 2008, Ocean Wave Energy: Current Status and Future Perspectives, Springer, Berlin, Germany

10. FALCÃO A.F. DE O., 2010, Wave energy utilization: a review of the technologies, Renewable and Sustainable Energy Reviews, Elsevier, 14, 3, 899-918

11. French M.J., 2006, On the difficulty of inventing an economical sea wave energy converter: a personal view, Journal of Engineering for the Maritime Environment, 220, 3, 149-155

12. Le Crom I., Brito-Melo A., Neumann F., Sarmento A., 2009, Numerical estimation of incident wave parameters based on the air pressure measurements in pico OWC plant, Proceedings of the 8th European Wave and Tidal Energy Conference, Uppsala, Sweden

13. Lucas J., Salter S.H., Cruz J., Taylor J.R.M., Bryden I., 2007, Performance optimisation of a modified Duck through optimal mass distribution, Proceedings of the rth European Wave and Tidal Energy Conference, Porto, Portugal

14. ŁAdyŻyńska-Kozdraś E., Koruba Z., 2012, Model of the final section of navigation of a self-guided missile steered by gyroscope, Journal of Theoretical and Applied Mechanics, 50, 2

15. Muetze A, Vining J.G., 2006, Ocean wave energy conversion - a survey, Proceedings of the ASME Conference Forty-First IAS Annual Meeting, Tampa, USA, 3, 1410-1417

16. Newman J., 1977, Marine Hydrodynamics, The MIT Press

17. Pizer D.J., Retzler C., Henderson R.M., Cowieson F.L., Shaw M.G., Dickens B., HART R., 2005, Pelamis WEC - Recent advances in the numerical and experimental modelling programme, Proceedings of the 6th European Wave and Tidal Energy Conference, Glasgow, UK

18. SALTER S.H., 1974, Wave power, Nature, 249, 720-724 
19. Sørensen H.C., Friss-Madsen E., 2010, Wave dragon from demonstration to market, Proceedings of the 3rd International Conference on Ocean Energy, Bilbao, Spain

20. Taylor J.R.M., Rea M., Rogers D.J., 2003, The Edinburgh curved tank, Proceedings of the 5th European Wave and Tidal Energy Conference, Cork, UK

21. Torre-Enciso Y., Marqués J., López de Aguileta L.I., 2010, Mutriku. Lessons learnt, Proceedings of the 3rd International Conference on Ocean Energy, Bilbao, Spain

22. Vicinanza D., Cappietti L., Contestabile P., 2009, Assessment of wave energy around Italy, Proceedings of the 8th European Wave and Tidal Conference, EWTEC

Manuscript received March 17, 2014; accepted for print August 27, 2014 